\title{
AN INTRINSIC ORDER-THEORETIC CHARACTERIZATION OF THE WEAK EXPECTATION PROPERTY
}

\author{
MARTINO LUPINI
}

\begin{abstract}
We prove the following characterization of the weak expectation property for operator systems in terms of Wittstock's matricial Riesz separation property: an operator system $S$ satisfies the weak expectation property if and only if $M_{q}(S)$ satisfies the matricial Riesz separation property for every $q \in \mathbb{N}$. This can be seen as the noncommutative analog of the characterization of simplex spaces among function systems in terms of the classical Riesz separation property.
\end{abstract}

\section{INTRODUCTION}

An operator system is a closed subspace of a unital $\mathrm{C}^{*}$-algebra that contains the unit and is invariant under the involution. Operator systems can be seen as the noncommutative analog of compact convex sets. Indeed, one can associate with any compact convex set the operator system $A(K) \subset C(K)$ of continuous affine complexvalued functions on $K$. The operator systems arising in this way are called function systems, and are precisely the operator systems that can be represented within an abelian unital $\mathrm{C}^{*}$-algebra. The study of operator systems goes as far back as the works of Arveson in the late 1960s [2, 3] and of Choi-Effros in the 1970s [10-12, 12, 13] from the 1970s. Since then, it has been pursued by many authors [4-6, 14, 18, 20-25, 32, 34, 39, 41, 42, 46-51]; see also the monographs $[17,43,44]$.

The program of studying "noncommutative order" within the framework of operator systems has been explicitly proposed by Effros in 1977 [15]. Towards this goal, in view of the key role that Choquet simplices play in classical convexity theory, it is important to understand what is the correct noncommutative analog of Choquet simplices. This can be a challenging problem, as many different equivalent characterization of Choquet simplices exist. Several of such characterizations admit natural noncommutative analogues, which are not obviously equivalent. It might therefore seem arbitrary to decide that any given one of such noncommutative analogues is the "right" notion of noncommutative Choquet simplex. However, it is arguable that, if such a noncommutative analogues turn out to be equivalent, at least under some generous assumptions, then the corresponding notion of "noncommutative Choquet simplex" would be sufficiently robust and, in a sense, the correct one.

First of all, in order to have hopes of a well-behaved theory, or at least of a theory where as many results as possible from the commutative case can be transferred, one should restrict to the class of exact operator systems. Indeed, (non)exactness has no analogue in the commutative setting, and any function system is automatically exact. The result of Junge and Pisier [33] that the class of finite-dimensional operator systems with respect to the completely bounded Banach-Mazur distance is not separable (unlike the class of finite-dimensional exact operator systems) also suggests that the class of nonexact of operator systems is too wild.

The main result of this paper is that, within the class of exact operator systems, three natural noncommutative analogues of the notion of Choquet simplex of very different nature- operator-theoretic, order-theoretic, and model-theoretic - are in fact equivalent. The first characterization of Choquet simplices that we consider can be formulated in terms of the completely positive approximation property. A compact convex set $K$ is a Choquet simplex if and only if the identity map of $A(K)$ is the pointwise limit of unital completely positive maps that factor through finite-dimensional injective operator systems (which in this case can be chosen to be function systems). This definition applies equally well to the noncommutative setting, yielding the important notion of nuclear operator systems. It is hard to understate the importance of nuclearity for the theory of operator algebras and operator systems. For instance, this notion plays a crucial role in the Choi-Effros solution to the completely positive lifting problem for $C^{*}$-algebras [10]. Several equivalent reformulations of nuclearity are already known; see for instance [31, 36, 37].

2000 Mathematics Subject Classification. Primary 47L25, 46L89; Secondary 46A55, 52A07.

Key words and phrases. Operator system, function system, weak expectation property, Riesz separation property, positively existentially closed, matrix sublinear functional.

The author was partially supported by the NSF Grant DMS-1600186. 
The second characterization of Choquet simplices that we consider is order-theoretic. In classical convexity, this can be stated in terms of the Riesz separation property for the space $A(K)$ as an ordered vector space [1]. A matricial version of the Riesz separation property for operator systems has been considered by Wittstock in [49-51]. This property, formulated in terms of the notion of matrix sublinear functional introduced therein, plays a crucial role in Wittstock's proof of the decomposition and extension theorems for completely bounded maps (obtained independently by Haagerup and by Paulsen). Along the way, Wittstock proved that for dual operator systems the matricial Riesz separation property is equivalent to injectivity [49, Satz 3.6]; see also [45, Theorem 2.4]. The main result of this paper is that one can, more generally, provide a similar intrinsic ordertheoretic characterization of nuclearity. Precisely, an exact operator system $S$ is nuclear if and only if, for every $q \in \mathbb{N}, M_{q}(S)$ endowed with its canonical operator system structure satisfies the matricial Riesz separation property.

In fact, our proof applies to arbitrary (not necessarily exact) operator systems, in which case it provides a characterization of the weak expectation property. Thus, an operator system $S$ satisfies the weak expectation property if and only if $M_{q}(S)$ satisfies the matricial Riesz separation property for every $q \in \mathbb{N}$. Other characterizations of the weak expectation property have been obtained in $[19,35]$ in the case of $\mathrm{C}^{*}$-algebras. Particularly, in [35, Theorem 7.4] the weak expectation property for $\mathrm{C}^{*}$-algebras is shown to be equivalent to the "complete tight Riesz interpolation property" introduced therein [35, Definition 7.1]. The complete tight Riesz interpolation property, while superficially similar, is very different in spirit from Wittstock's matricial Riesz separation property. For instance, while the matricial Riesz separation property is an intrinsic notion, which only refers to the given operator system $S$ itself and its matricial positive cones, the complete tight Riesz interpolation property is defined in terms of a concrete realization of $S$ as a space of operators. While different in spirit, these notions turn out to be in fact equivalent, as we deduce from our main results.

The third and last characterization of Choquet simplices that we consider is model-theoretic. It asserts that a compact convex set $K$ is a Choquet simplex if and only if the corresponding function system $A(K)$ is positively existentially closed within the class of function systems [40, Subsection 6.4]. This means that if one can find in a function system $V$ containing $A(K)$ a certain configuration defined in terms of conditions of the form $\|p(\bar{x})\|<r$, where $p(\bar{x})$ is a degree $1^{*}$-polynomial with coefficients from $A(K)$ and $r \in \mathbb{R}$, then one can also find such a configuration within $A(K)$. We show that, similarly, an operator system satisfies the weak expectation property if and only if it is positively existentially closed within the class of operator systems. This is defined as above, where one considers configurations defined in terms of norms of matrices of degree 1 *-polynomials; see Section 3. In the case of $\mathrm{C}^{*}$-algebras, this recovers a result of Goldbring and Sinclair from [29]. The model-theoretic notion of positively existentially closed structure, and the related notion of positively existential embedding, have recently found several applications to the study of $\mathrm{C}^{*}$-algebras and $\mathrm{C}^{*}$-dynamics, as in the work of Goldbring and Sinclair on the Kirchberg embedding problem [30] and the works of Barlak and Szabo [7] and Gardella and the author [27] providing a unified approach to several preservation results in $\mathrm{C}^{*}$-dynamics for actions of compact groups with finite Rokhlin dimension. This perspective has also been used to generalize these preservation results to the case of compact quantum groups [8, 26].

The rest of this paper is divided into two sections. In Section 2 we recall some terminology concerning "noncommutative order" as introduced by Wittstock, including matrix sublinear functionals and the matricial Riesz separation property, and then we prove the order-theoretic characterization of the weak expectation property mentioned above. In Section 3 we describe in more detail the notion of positively existentially closed operator system, and prove its equivalence with the weak expectation property.

In the following, we adopt standard notations from the theory of operator systems. Particularly, we denote operator systems as $S$ or $T$. We let $M_{q}(S)$ be the space of $q \times q$ matrices with entries in $S$, endowed with its canonical operator system structure obtained from the identification with $M_{n}(\mathbb{C}) \otimes S$. If $\phi: S \rightarrow T$ is a linear map between operator systems, and $q \in \mathbb{N}$, then we let $\phi_{q}: M_{q}(S) \rightarrow M_{q}(T)$ be the corresponding amplification defined by $\phi_{q}\left(\left[x_{i j}\right]\right)=\left[\phi\left(x_{i j}\right)\right]$. The completely bounded norm $\|\phi\|_{\mathrm{cb}}$ of $\phi$ is defined to be the supremum of $\left\|\phi_{q}\right\|$ for $q \in \mathbb{N}$. We denote by $\operatorname{Ball}(S)$ the closed unit ball of the operator system $S$.

\section{AN ORDER-THEORETIC CHARACTERIZATION OF THE WEAK EXPECTATION PROPERTY}

2.1. The weak expectation property. Let $S$ be an operator system, and $S^{* *}$ be its second dual. We endow $S^{* *}$ with its canonical operator system structure, and identify $S$ with its image under the canonical inclusion inside $S^{* *}$. The notion of weak expectation property for $S$ has been introduced in [36, Definition 6.4] and [16, Section 4]. 
Definition 2.1. The operator system $S$ has the weak expectation property if for any inclusion of operator systems $E \subset F$ and unital completely positive map $\phi: E \rightarrow S$, there exists a unital completely positive map $\tilde{\phi}: F \rightarrow S^{* *}$ extending $\phi$.

The following characterization of the weak expectation property is a consequence of [11, Theorem 6.1].

Lemma 2.2. Suppose that $S$ is an operator system. Then $S$ has satisfies the weak expectation property if and only if for every $d \in \mathbb{N}$, inclusion of operator systems $E \subset M_{d}(\mathbb{C})$ and unital completely positive map $\phi: E \rightarrow S$, there exists a unital completely positive map $\tilde{\phi}: M_{d}(\mathbb{C}) \rightarrow S^{* *}$ extending $\phi$.

The following characterization of the weak expectation property is an consequence of Lemma 2.2 via a standard approximation argument; see also [29, Proposition 4.12] and [9, Proposition 2.3.8]. We present the details for the sake of completeness.

Proposition 2.3. Suppose that $S$ is an operator system. The following statements are equivalent:

(1) $S$ satisfies the weak expectation property;

(2) for any $d \in \mathbb{N}$, inclusion of operator systems $E \subset M_{d}(\mathbb{C})$, unital completely positive map $\phi: E \rightarrow S$, and $\varepsilon>0$, there exists a linear map $\psi: M_{d}(\mathbb{C}) \rightarrow S$ such that $\left\|\psi_{d}\right\| \leq 1+\varepsilon,\|\psi(1)-1\| \leq \varepsilon$, and $\left\|\left.\psi\right|_{E}-\phi\right\| \leq \varepsilon$

(3) for any $d \in \mathbb{N}$, inclusion of operator systems $E \subset M_{d}(\mathbb{C})$, unital completely positive map $\phi: E \rightarrow S$, and $\varepsilon>0$, there exists a unital completely positive map $\psi: M_{d}(\mathbb{C}) \rightarrow S$ such that $\left\|\left.\psi\right|_{E}-\phi\right\|<\varepsilon$.

Proof. The fact that (3) implies (1) is easily seen by taking a $\sigma\left(S^{* *}, S^{*}\right)$-limit. The implication (2) $\Rightarrow(3)$ is a standard approximation argument; see [28, Lemma 4.3]. We prove the remaining implication $(1) \Rightarrow(2)$.

Fix $d \in \mathbb{N}$, an operator system $E \subset M_{d}(\mathbb{C})$, a unital completely positive map $\phi: E \rightarrow S$, and $\varepsilon>0$. By assumption there exists a unital completely positive map $\tilde{\phi}: M_{d}(\mathbb{C}) \rightarrow S^{* *}$ such that $\left.\tilde{\phi}\right|_{E}=\phi$. Consider the correspondence between completely positive maps $\psi: M_{d}(\mathbb{C}) \rightarrow S^{* *}$ and positive elements $a_{\psi}=\left[\psi\left(e_{i j}\right)\right]$ of $M_{d}\left(S^{* *}\right)$. Since $M_{d}(S)$ is $\sigma\left(S^{* *}, S^{*}\right)$-weakly dense in $M_{d}\left(S^{* *}\right)$, there exists a net $\left(\psi_{\lambda}\right)$ of completely positive maps $\psi_{\lambda}: M_{d}(\mathbb{C}) \rightarrow S$ such that $\psi_{\lambda}(x) \rightarrow \phi(x)$ in the $\sigma\left(S^{* *}, S^{*}\right)$-topology for every $x \in M_{d}(\mathbb{C})$. Fix $x_{1}, \ldots, x_{n} \in E$ and $\delta>0$. Consider the net

$$
\eta_{\lambda}=\left(\psi_{\lambda}\left(x_{1}\right)-\phi\left(x_{1}\right), \ldots, \psi_{\lambda}\left(x_{n}\right)-\phi\left(x_{n}\right), \psi_{\lambda}(1)-1\right)
$$

in $S \oplus \cdots \oplus S$. We have that $\left(\eta_{\lambda}\right)$ converges to 0 weakly. It follows from the Hahn-Banach theorem that the norm closure of the convex hull of $\left(\eta_{\lambda}\right)$ coincides with the weak closure of the convex hull of $\left(\eta_{\lambda}\right)$. Therefore 0 belongs to the norm closure of the convex hull of $\left(\eta_{\lambda}\right)$. Therefore there exists a convex combinations $\bar{\psi}$ of the $\psi_{\lambda}$ 's such that $\left\|\bar{\psi}\left(x_{i}\right)-\phi\left(x_{i}\right)\right\|<\delta$ for $i=1,2, \ldots, n$ and $\|\bar{\psi}(1)-1\|<\delta$. Since $\bar{\psi}$ is a convex combination of the $\psi_{\lambda}$ 's, we have that $\bar{\psi}$ is completely positive, and in particular $\|\bar{\psi}\|_{c b}=\|\bar{\psi}(1)\| \leq 1+\delta$. By [40, Lemma 8.1] there exists a unital completely positive map $\psi: M_{d}(\mathbb{C}) \rightarrow S$ such that $\|\psi-\bar{\psi}\| \leq 2 d^{2} \delta$. By choosing $\delta>0$ small enough, one can ensure that $\left\|\left.\psi\right|_{E}-\phi\right\| \leq \varepsilon$. This concludes the proof.

Recall that an operator system $S$ is exact if for any finite-dimensional subspace $E$ of $S$ and $\varepsilon>0$ there exist $d \in \mathbb{N}$, a subspace $F$ of $M_{d}(\mathbb{C})$, and unital completely positive maps $\phi: E \rightarrow F$ and $\psi: F \rightarrow E$ such that $\left\|\psi \circ \phi-\mathrm{id}_{E}\right\|<\varepsilon$ and $\left\|\psi \circ \phi-\mathrm{id}_{F}\right\|<\varepsilon$. An operator system $S$ is nuclear if it satisfies the completely positive approximation property, namely there exists nets $\left(\rho_{i}\right),\left(\gamma_{i}\right)$ of unital completely positive maps $\gamma_{i}: S \rightarrow M_{d_{i}}(\mathbb{C})$ and $\rho_{i}: M_{d_{i}}(\mathbb{C}) \rightarrow S$ such that $\left\|\left(\rho_{i} \circ \gamma_{i}\right)(x)-x\right\| \rightarrow 0$ for every $x \in S$. It follows from Proposition 2.3 that an operator system is nuclear if and only if it is exact and it satisfies the weak expectation property.

2.2. Matrix sublinear functionals and noncommutative order. We recall in these sections some notions about matrix sublinear functionals and noncommutative order introduced by Wittstock in [49-51]; see also $[15,45]$.

Suppose that $V$ is an ordered real vector spaces. For subsets $A, B$ of $V$, we let $A \preccurlyeq B$ if and only if for every $b \in B$ there exists $a \in A$ such that $a \leq b$; see [49, Definition 2.1.1]. We identify an element $a$ of $V$ with the corresponding singleton. Consistently, we write $a \preccurlyeq B$ if $a \leq b$ for every $b \in B$. A *-vector space is a complex vector space $V$ endowed with a conjugate-linear map $V \rightarrow V, x \mapsto x^{*}$ satisfying $\left(x^{*}\right)^{*}=x$. The real subspace of elements satisfying $x^{*}=x$ will be denoted by $V_{\text {sa. }}$. The space $M_{n}(V)$ of $n \times n$ matrices with entries in $V$ has a canonical $*$-vector space structure obtained by setting $\left[x_{i j}\right]^{*}=\left[x_{j i}^{*}\right]$. A $*$-vector space is ordered if it is endowed with a distinguished proper convex cone $V^{+} \subset V_{\text {sa }}$, and matrix ordered if it is endowed with distinguished proper convex cones $M_{n}(V)^{+} \subset M_{n}(V)_{\text {sa }}$ such that $\gamma^{*} M_{n}(V)^{+} \gamma \subset M_{m}(V)^{+}$for $n, m \in \mathbb{N}$ and $\gamma \in M_{n, m}(\mathbb{C})$. Any operator system is endowed with a canonical matrix ordered $*$-vector space structure.

The notion of matrix sublinear functional has been introduced by Wittstock in [49, Definition 2.1.1]. 
Definition 2.4. Let $V$ be a $*$-vector space and $W$ be an operator system. Suppose that $\theta$ is a sequence $\left(\theta_{n}\right)_{n \in \mathbb{N}}$, where $\theta_{n}$ is a function that assigns to any element $v$ of $M_{n}(V)_{\text {sa }}$ a subset $\theta_{n}(v)$ of $M_{n}(W)_{\text {sa }}$. Then $\theta: V \rightarrow W$ is a matrix sublinear functional if, for every $u, v \in M_{n}(V)_{\mathrm{sa}}, \gamma \in M_{n, m}(\mathbb{C})$, and $n, m \in \mathbb{N}$, one has the following:

(1) $\theta_{n}(v)$ is nonempty,

(2) $\theta_{n}(u+v) \preccurlyeq \theta_{n}(u)+\theta_{n}(v)$,

(3) $0 \preccurlyeq \theta_{n}(0)$,

(4) $\theta_{m}\left(\gamma^{*} v \gamma\right) \preccurlyeq \gamma^{*} \theta_{n}(v) \gamma$

We say that a matrix sublinear functional $\theta: V \rightarrow W$ is completely positive if $0 \preccurlyeq \theta_{n}(v)$ for every $n \in \mathbb{N}$ and $v \in M_{n}(V)_{\mathrm{sa}}$.

Further properties of matrix sublinear functionals are listed in [49, Lemma 2.1.3]. Observe that a (completely positive) selfadjoint linear map $\phi: V \rightarrow W$ can be regarded as a (completely positive) matrix sublinear functional in the obvious say.

Suppose that $S$ is an operator system, and $\alpha \in M_{n}(\mathbb{C})_{\text {sa. }}$. An element $x$ of $M_{n}(S)_{\text {sa }}$ is $\alpha$-positive if, whenever $\gamma_{1}, \ldots, \gamma_{\ell} \in M_{n, m}(\mathbb{C})$ for $\ell, m \in \mathbb{N}$ are such that $\gamma_{1}^{*} \alpha \gamma_{1}+\cdots+\gamma_{\ell}^{*} \alpha \gamma_{\ell}=0$, one has that $\gamma_{1}^{*} x \gamma_{1}+\cdots+\gamma_{\ell}^{*} x \gamma_{\ell} \geq 0$ [49, Definition 3.1(a)]; see also [45, Definition 2.2]. We say that $x \in M_{n}(S)_{\mathrm{sa}}$ is strictly $\alpha$-positive if, whenever $\gamma_{1}, \ldots, \gamma_{\ell} \in M_{n, m}(\mathbb{C})$ for $\ell, m \in \mathbb{N}$ are such that $\gamma_{1}^{*} \alpha \gamma_{1}+\cdots+\gamma_{\ell}^{*} \alpha \gamma_{\ell} \geq 0$ one has that $\gamma_{1}^{*} x \gamma_{1}+\cdots+\gamma_{\ell}^{*} x \gamma_{\ell} \geq 0$. An element $v$ of $S_{\text {sa }}$ is a lower $\alpha$-bound for $x$ if $v \otimes \alpha \leq x$ [49, Definition 3.1(b)]; see also [45, Definition 2.2].

Remark 2.5. Suppose that $\alpha_{i} \in M_{n_{i}}(\mathbb{C})_{\mathrm{sa}}$ and $x_{i} \in M_{n_{i}}(S)_{\mathrm{sa}}$ is (strictly) $\alpha_{i}$-positive for $i=1,2, \ldots, n$. Set $n:=n_{1}+\cdots+n_{\ell}, \alpha:=\alpha_{1} \oplus \cdots \oplus \alpha_{\ell} \in M_{n}(\mathbb{C})_{\mathrm{sa}}$, and $x:=x_{1} \oplus \cdots \oplus x_{\ell} \in M_{n}(S)_{\mathrm{sa}}$. Then $x$ is (strictly) $\alpha$-positive.

We let $\sigma_{m, n}$ be the matrix $1_{m} \oplus\left(-1_{n}\right) \in M_{m+n}(\mathbb{C})$, where $1_{d} \in M_{d}(\mathbb{C})$ is the $d \times d$ identity matrix for $d \in \mathbb{N}$. The matricial Riesz separation property has been introduced by Wittstock in [49, Definition 3.1]; see also [45, Definition 2.2].

Definition 2.6 (Wittstock). An operator system $S$ satisfies the matricial Riesz separation property if for every $n \in \mathbb{N}$, every $\sigma_{n, n}$-positive $x \in M_{2 n}(S)_{\text {sa }}$ has a lower $\sigma_{n, n}$-bound $v \in S_{\text {sa }}$.

The following notion has also been considered by Wittstock in [51, Theorem 2.3] under the name of matricial Riesz separation property. To distinguish it from the property given by Definition 2.6, we call it the positive matricial Riesz separation property.

Definition 2.7. An operator system $S$ satisfies the positive matricial Riesz separation property if for every $n \in \mathbb{N}$, every strictly $\sigma_{n, n}$-positive $x \in M_{2 n}(S)_{\text {sa }}$ has a lower $\sigma_{n, n}$-bound $v \in S^{+}$.

It is remarked at the end of Section 2.1 in [51, Theorem 2.3] that "it does not seem to be obvious that both properties [considered in Definition 2.6 and Definition 2.7] are equivalent".

The matricial Riesz separation property can be seen as the noncommutative analog of the Riesz separation property for an ordered vector space. Recall that a real ordered vector space $V$ satisfies the Riesz separation property if for any $n \in \mathbb{N}, x_{1}, \ldots, x_{n}, y_{1}, \ldots, y_{n} \in V$ such that $x_{i} \leq y_{j}$ for $i, j \in\{1,2, \ldots, n\}$ there exists $z \in V$ such that $x_{i} \leq z \leq y_{j}$ for $i, j \in\{1,2, \ldots, n\}$. We say that a $*$-vector space $V$ satisfies the Riesz separation property if $V_{\text {sa }}$ does.

Suppose now that $S$ is a function system, i.e. an operator system that can be represented inside a commutative $\mathrm{C}^{*}$-algebra. In this case, by the Kadison representation theorem [1, Theorem II.1.8], $S$ is unitally completely order isomorphic to the space $A(K)$ of complex-valued continuous affine functions on $K$, where $K$ is the state space of $S$ endowed with the w*-topology. It is well known that in this case $A(K)$ satisfies the Riesz separation property if and only if $K$ is a Choquet simplex [1, Corollary II.3.11]. It is proved in [51, Proposition 2.1] that $A(K)$ satisfies the Riesz separation property if and only if it satisfies the positive matricial Riesz separation property from Definition 2.7. In fact, one can directly prove the following.

Proposition 2.8. Suppose that $S=A(K)$ is a function system with state space $K$. The following assertions are equivalent.

(1) S satisfies the Riesz separation property;

(2) $S$ satisfies the matricial Riesz separation property;

(3) $S$ satisfies the positive matricial Ries separation property;

(4) $M_{q}(S)$ satisfies the matricial Riesz separation property for every $q \in \mathbb{N}$;

(5) $M_{q}(S)$ satisfies the positive matricial Riesz separation property for every $q \in \mathbb{N}$. 
Proof. $(1) \Rightarrow(4)$ : Suppose that $S$ satisfies the Riesz separation property, in which case $K$ is a Choquet simplex by [1, Corollary II.3.11]. Fix $q, n \in \mathbb{N}$, and a $\sigma_{n, n}$-positive element $a \in M_{2 n}\left(M_{q}(S)\right)_{\text {sa }}$. Observe that $M_{2 n}\left(M_{q}(S)\right)$ can be identified with the space of $M_{2 n}\left(M_{q}(\mathbb{C})\right.$ )-valued continuous affine functions on $K$. Consistently, we regard $a$ as a function from $K$ to $M_{2 n}\left(M_{q}(\mathbb{C})\right)_{\text {sa }}$, and denote by $a(p)$ the value of $a$ at $p \in K$. Fix $p \in K$. Since $a$ is $\sigma_{n, n}$-positive, $a(p) \in M_{2 n}\left(M_{q}(\mathbb{C})\right)_{\text {sa }}$ is $\sigma_{n, n}$-positive. Therefore by [51, Theorem 2.4] there exists $\alpha \in M_{q}(\mathbb{C})_{\text {sa }}$ such that $\alpha \otimes \sigma_{n, n} \leq a(p)$. This shows that the set

$$
\Phi(p):=\left\{\alpha \in M_{q}(\mathbb{C})_{\mathrm{sa}}: \alpha \otimes \sigma_{n, n} \leq a(p)\right\}
$$

is a nonempty closed convex subset of $M_{q}(\mathbb{C})_{\text {sa }}$. Since $a$ is affine, the assignment $p \mapsto \Phi(p)$ satisfies

$$
\Phi(t p+(1-t) q) \supset t \Phi(p)+(1-t) \Phi(q) .
$$

Furthermore, since $a$ is continuous, for any open subset $U$ of $M_{q}(\mathbb{C})_{\text {sa }}$ the set

$$
\{p \in K: \Phi(p) \cap U \neq \varnothing\}
$$

is open. Therefore by Lazar's selection theorem [38, Theorem 3.1] there exists $b \in A\left(K, M_{q}(\mathbb{C})_{\text {sa }}\right)=M_{q}(S)_{\text {sa }}$ such that $b(p) \in \Phi(p)$ for every $p \in K$ or, equivalently, $b \otimes \sigma_{n, n} \leq a$. This concludes the proof.

The proof of the implication $(1) \Rightarrow(5)$ is entirely analogous to the proof of the implication $(1) \Rightarrow(4)$, where one replaces [51, Theorem 2.4] with [51, Lemma 2.2]. The implication $(3) \Rightarrow(1)$ is observed in [51, Section 2.2], and the implication $(2) \Rightarrow(1)$ is analogous to the implication $(3) \Rightarrow(1)$. The rest of the implications are trivial.

It is well known that, in the commutative case, the Riesz separation property is equivalent to the approximate Riesz separation property; see [1, Corollary II.3.11]. The natural noncommutative analog of this fact holds as well.

Lemma 2.9. Let $S$ be an operator system. Suppose that for every $n \in \mathbb{N}$, every $\sigma_{n, n}$-positive $x \in M_{2 n}(S)_{\text {sa }}$, and $\varepsilon>0$, the element $x+\varepsilon 1_{n} \in M_{2 n}(S)_{\text {sa }}$ has a lower $\sigma_{n, n}$-bound $v \in M_{q}(S)_{\text {sa }}$. Then $S$ satisfies the matricial Riesz separation property.

Proof. Using Remark 2.5, it is easy to see that $S$ satisfies the following property: for every $q, n_{1}, \ldots, n_{\ell} \in \mathbb{N}$, $\sigma_{n_{i}, n_{i}}$-positive $x_{i} \in M_{2 n_{i}}(S)_{\mathrm{sa}}$, and $\varepsilon>0$, there exists $v \in S_{\mathrm{sa}}$ such that $v \otimes \sigma_{n_{i}, n_{i}} \leq x_{i}+\varepsilon 1_{n_{i}}$ for $i=1,2, \ldots, \ell$. Indeed, one can consider $n:=n_{1}+\cdots+n_{\ell}, \alpha:=\sigma_{n_{1}, n_{1}} \oplus \cdots \oplus \sigma_{n_{\ell}, n_{\ell}} \in M_{2 n}(\mathbb{C})_{\text {sa }}$, and $x:=x_{1} \oplus \cdots \oplus x_{\ell} \in$ $M_{2 n}(S)_{\text {sa }}$. Then $x$ is $\alpha$-positive, and $\alpha$ is unitarily conjugate to $\sigma_{2 n, 2 n}$. Therefore by assumption there exists $v \in S_{\mathrm{sa}}$ such that $v \otimes \alpha \leq x+\varepsilon 1_{2 n}$. Hence $v \otimes \sigma_{n_{i}, n_{i}} \leq x_{i}+\varepsilon 1_{n_{i}}$ for $i=1,2, \ldots, \ell$.

Fix now $n \in \mathbb{N}$ and a $\sigma_{n, n}$-positive element $x \in M_{2 n}(S)$. We want to show that there exists $v \in S_{\text {sa }}$ such that $v \otimes \sigma_{n, n} \leq x$. By assumption, there exists a $v_{0} \in S_{\text {sa }}$ such that $v_{0} \otimes \sigma_{n, n} \leq x+1_{n}$. Observe now that $v_{0} \otimes \sigma_{1,1}$ is $\sigma_{1,1}$-positive. Therefore by assumption there exists $v_{1} \in S_{\text {sa }}$ such that $v_{1} \otimes \sigma_{n, n} \leq x+2^{-1} 1_{n}$ and $v_{1} \otimes \sigma_{1,1} \leq v_{0} \otimes \sigma_{1,1}+2^{-1} 1_{2}$. Proceeding in this way, one can define at the $k$-th step an element $v_{k} \in S_{\text {sa }}$ such that $v_{k} \otimes \sigma_{n, n} \leq x+2^{-k} 1_{n}$ and $v_{k} \otimes \sigma_{1,1} \leq v_{k-1} \otimes \sigma_{1,1}+2^{-k} 1_{2}$. Thus $\left(v_{k}\right)$ is a Cauchy sequence in $M_{q}(S)_{\text {sa }}$ converging to an element $v$ of $M_{q}(S)_{\text {sa }}$ such that $v \otimes \sigma_{n, n} \leq x$.

The same argument applies to the case of the positive matricial Riesz separation property, and gives the following lemma.

Lemma 2.10. Let $S$ be an operator system. Suppose that for every $n \in \mathbb{N}$, every strictly $\sigma_{n, n}$-positive $x \in M_{2 n}(S)_{\mathrm{sa}}$, and $\varepsilon>0$, the element $x+\varepsilon 1_{n} \in M_{2 n}(S)_{\text {sa }}$ has a lower $\sigma_{n, n}$-bound $v \in M_{q}(S)^{+}$. Then $S$ satisfies the positive matricial Riesz separation property.

2.3. The proof of the main theorem. The goal of this subsection is to prove the following characterization of the weak expectation property for operator systems in terms of Wittstock's matricial Riesz separation property.

Theorem 2.11. Suppose that $S$ is an operator system. The following assertions are equivalent:

(1) $S$ satisfies the weak expectation property;

(2) $M_{q}(S)$ satisfies the matricial Riesz separation property for every $q \in \mathbb{N}$;

(3) $M_{q}(S)$ satisfies the positive matricial Riesz separation property for every $q \in \mathbb{N}$;

(4) For every $q \in \mathbb{N}$ and matrix sublinear functional $\theta: \mathbb{C} \rightarrow M_{q}(S)$ there exists $v \in M_{q}\left(S^{* *}\right)_{\mathrm{sa}}$ such that $v \otimes \alpha \preccurlyeq \theta_{n}(\alpha)$ for every $n \in \mathbb{N}$ and $\alpha \in M_{n}(\mathbb{C})_{\mathrm{sa}} ;$

(5) For every $q \in \mathbb{N}$ and completely positive matrix sublinear functional $\theta: \mathbb{C} \rightarrow M_{q}(S)$ there exists $v \in M_{q}\left(S^{* *}\right)^{+}$such that $v \otimes \alpha \preccurlyeq \theta_{n}(\alpha)$ for every $n \in \mathbb{N}$ and $\alpha \in M_{n}(\mathbb{C})_{\mathrm{sa}} ;$

(6) For every $q \in \mathbb{N}$, *-vector space $V$, selfadjoint subspace $W \subset V$, matrix sublinear functional $\theta: V \rightarrow$ $M_{q}(S)$, and selfadjoint linear map $\phi_{0}: W \rightarrow M_{q}(S)$ such that $\left.\phi_{0} \preccurlyeq \theta\right|_{W}$, there exists a selfadjoint linear map $\phi: V \rightarrow M_{q}\left(S^{* *}\right)$ such that $\phi \preccurlyeq \theta$ and $\left.\phi\right|_{W}=\phi_{0}$. 
Proof. $(1) \Rightarrow(2)$ : Suppose that $S$ satisfies the weak expectation property. Fix $q \in \mathbb{N}$. We verify that $M_{q}(S)$ satisfies the conditions of Lemma 2.9. Fix $n \in \mathbb{N}$, a $\sigma_{n, n}$-positive $x \in M_{2 n}\left(M_{q}(S)\right)_{\text {sa }}$, and $\varepsilon>0$. Fix a unital complete order embedding $\eta: S \rightarrow B(H)$. Since $S$ satisfies the weak expectation property, there exists a unital completely positive map $\phi: B(H) \rightarrow S^{* *}$ such that $\phi \circ \eta$ is the inclusion map of $S$ in $S^{* *}$. By [51, Theorem 2.4], $M_{q}(B(H)) \cong B(H \oplus \cdots \oplus H)$ satisfies the Riesz separation property. Therefore there exists $w \in M_{q}(B(H))_{\text {sa }}$ such $w \otimes \sigma_{n, n} \leq \eta_{q}(x)$. Thus $v:=(\phi \circ \eta)_{q}(w)$ is an element of $M_{q}\left(S^{* *}\right)_{\text {sa }}$ such that $v \otimes \sigma_{n, n} \leq x$. By $\sigma\left(S^{* *}, S^{*}\right)$-density of $S$ in $S^{* *}$ together with a convexity argument as in the proof of Proposition 2.3, one can now find $v \in M_{q}(S)_{\text {sa }}$ such that $v \otimes \sigma_{n, n} \leq x+\varepsilon 1_{n}$.

$(1) \Rightarrow(3)$ : This is the same as $(1) \Rightarrow(2)$, using [51, Theorem 2.3] instead of [51, Theorem 2.4] and Lemma 2.10 instead of Lemma 2.9.

$(2) \Rightarrow(4)$ : The proof of this implication is analogous to the proof of $[49$, Lemma 3.4]. Suppose that $\theta: \mathbb{C} \rightarrow$ $M_{q}(S)$ is a matrix sublinear functional. For $n \in \mathbb{N}$ and $\alpha \in M_{n}(\mathbb{C})_{\text {sa }}$, set

$$
L(\alpha):=\left\{r \in M_{q}\left(S^{* *}\right)_{\mathrm{sa}}: r \otimes \alpha \preccurlyeq \theta_{n}(\alpha)\right\} .
$$

If $m \in \mathbb{N}$ and $\gamma_{1}, \ldots, \gamma_{\ell} \in M_{n, m}(\mathbb{C})$ are such that $\gamma_{1}^{*} \alpha \gamma_{1}+\cdots+\gamma_{\ell}^{*} \alpha \gamma_{\ell}=0$, then

$$
0 \preccurlyeq \theta_{m}(0)=\theta_{m}\left(\gamma_{1}^{*} \alpha \gamma_{1}+\cdots+\gamma_{\ell}^{*} \alpha \gamma_{\ell}\right) \preccurlyeq \gamma_{1}^{*} \theta_{n}(\alpha) \gamma_{1}+\cdots+\gamma_{\ell}^{*} \theta_{n}(\alpha) \gamma_{\ell} \text {. }
$$

This shows that $\theta_{n}(\alpha) \subset M_{n}\left(M_{q}(S)\right)_{\text {sa }}$ is $\alpha$-positive. Set $L_{m, n}:=L\left(\sigma_{m, n}\right)$ for $m, n \in \mathbb{N}$. Observe that $L_{n, n} \subset L_{k, \ell}$ for $0 \leq k, \ell \leq n$, and $L_{n, n}$ is nonempty by assumption. If $\alpha \in M_{n}(\mathbb{C})_{\text {sa }}$, then $L(\alpha) \subset L_{k, \ell}$ for some $k, \ell \in \mathbb{N}$. Indeed, if $\alpha$ is invertible, then $\alpha=\beta^{*} \sigma_{k, \ell} \beta$ for some $\beta \in M_{n}(\mathbb{C})$ invertible and $k, \ell \in \mathbb{N}$. Thus if $r \in L_{k, \ell}$ then by [49, Lemma 2.1.3(f)]

$$
r \otimes \alpha=\beta^{*}\left(r \otimes \sigma_{k, \ell}\right) \beta \preccurlyeq \beta^{*} \theta_{n}\left(\sigma_{k, \ell}\right) \beta \preccurlyeq \theta_{n}\left(\beta^{*} \sigma_{k, \ell} \beta\right)=\theta_{n}(\alpha) .
$$

When $\alpha$ is not necessarily invertible, one can replace $\alpha$ with $\alpha+\delta 1_{n}$ for $\delta>0$ and then take the limit $\delta \rightarrow 0$. Therefore we have

$$
\bigcap_{n \in \mathbb{N}} L_{n, n}=\bigcap_{n \in \mathbb{N}} \bigcap_{\alpha \in M_{n}(\mathbb{C})} L(\alpha) .
$$

Such an intersection is nonempty by $\sigma\left(S^{* *}, S^{*}\right)$-compactness of the order intervals in $M_{q}\left(S^{* *}\right)^{+}$. Any element in such an intersection satisfies the desired conclusions.

$(3) \Rightarrow(5)$ : This is the same as the implication $(2) \Rightarrow(4)$.

$(4) \Rightarrow(1)$ : Suppose that $q \in \mathbb{N}, E \subset M_{q}(\mathbb{C})$, and $\phi: E \rightarrow S$ is a unital completely positive map. Let $e_{i j}$ be the matrix units of $M_{q}(\mathbb{C})$, and $e=\left[e_{i j}\right] \in M_{q}\left(M_{q}(\mathbb{C})\right)^{+}$be the Choi matrix. Define a matrix sublinear functional $\rho: M_{q}(\mathbb{C}) \rightarrow S$ by setting, for $n \in \mathbb{N}$ and $\alpha \in M_{n}\left(M_{q}(\mathbb{C})\right)_{\text {sa }}$,

$$
\rho_{n}(\alpha)=\left\{\phi_{n}(\beta): \beta \in M_{n}(E), \beta \geq \alpha\right\} \subset M_{n}(S)_{\mathrm{sa}} .
$$

It is not difficult to verify that $\rho$ is indeed a matrix sublinear functional. Define now a matrix sublinear functional $\theta: \mathbb{C} \rightarrow M_{q}(S)$ by

$$
\theta_{n}(\alpha)=\rho_{n q}(e \otimes \alpha) \subset M_{n}\left(M_{q}(S)\right)_{\mathrm{sa}} .
$$

By assumption, there exists $v \in M_{q}\left(S^{* *}\right)_{\text {sa }}$ such that $v \otimes \alpha \preccurlyeq \theta_{n}(\alpha)$ for every $n \in \mathbb{N}$ and $\alpha \in M_{n}(\mathbb{C})$. In particular we have that $-v \preccurlyeq \theta_{n}(-1)=\rho_{q}(-e) \preccurlyeq \phi_{q}(-e) \leq 0$ and hence $v \in M_{q}\left(S^{* *}\right)^{+}$. Consider now the completely positive map $\tilde{\phi}: M_{q}(\mathbb{C}) \rightarrow S^{* *}$ such that $\tilde{\phi}_{q}(e)=v$. For $\alpha \in E$ we have that

$$
\tilde{\phi}_{q n}(e \otimes \alpha)=v \otimes \alpha \preccurlyeq \theta_{n}(\alpha)=\rho_{n q}(e \otimes \alpha) \preccurlyeq \phi_{q n}(e \otimes \alpha) .
$$

For $i=1,2, \ldots, q$, let $\xi_{i} \in \mathbb{C}^{q}$ be the column vector whose only nonzero entry is a 1 in the $i$-th row, and then set

$$
\xi=\left[\begin{array}{c}
\xi_{1} \\
\xi_{2} \\
\vdots \\
\xi_{q}
\end{array}\right] \in \mathbb{C}^{q^{2}}
$$

Then we have that

$$
\tilde{\phi}(\alpha)=\xi^{*} \tilde{\phi}_{q n}(e \otimes \alpha) \xi \leq \xi^{*} \phi_{q n}(e \otimes \alpha) \xi=\phi(\alpha)
$$

This shows that $\left.\tilde{\phi}\right|_{E}=\phi$.

$(5) \Rightarrow(1)$ : This is the same as $(4) \Rightarrow(1)$.

$(1) \Rightarrow(6)$ : Consider a unital complete order embedding $\eta: S \rightarrow B(H)$. By [49, Theorem 2.3.1] there exists a selfadjoint linear map $\psi: V \rightarrow M_{q}(B(H))$ such that $\left.\psi\right|_{W}=\eta_{q} \circ \phi_{0}$ and $\psi \preccurlyeq \eta_{q} \circ \theta$. Since $S$ satisfies the weak 
expectation property, there exists a unital completely positive map $\rho: S \rightarrow S^{* *}$ such that $\eta \circ \rho: S \rightarrow S^{* *}$ is the inclusion map. Therefore the map $\phi:=\rho_{q} \circ \eta$ satisfies the desired conclusions.

$(6) \Rightarrow(4)$ : Obvious.

Suppose now that $S$ is a function system, in which case $S \cong A(K)$ where $K$ is the state space of $S$. In this case, $S$ satisfies the weak expectation property if and only if it is nuclear, which in turn is equivalent to the assertion that $K$ is a simplex. Therefore in view of Proposition 2.8, Theorem 2.11 can be seen as the noncommutative analog of the fact that a compact convex set $K$ is a simplex if and only if $A(K)$ satisfies the Riesz separation property.

\section{THE WEAK EXPECTATION PROPERTY AND MODEL THEORY}

3.1. Positively existentially closed operator systems. We now recall some notions from the logic for metric structures, in the specific setting of operator systems. Let us say that a degree 1 matrix *-polynomial is an expression $p\left(x_{1}, \ldots, x_{n}\right)$ of the form

$$
\alpha_{1}^{*} x_{1} \beta_{1}+\cdots+\alpha_{n}^{*} x_{n} \beta_{n}+\gamma_{1}^{*} 1 \delta_{1}+\cdots+\gamma_{n}^{*} 1 \delta_{n}
$$

where $\alpha_{i}, \beta_{i}, \gamma_{i}, \delta_{i}$ are scalar matrices of size $1 \times d$ for some $d \in \mathbb{N}$. Let $S$ be an operator system, and $a_{1}, \ldots, a_{n}$ be elements of $S$. Identifying 1 in $p\left(x_{1}, \ldots, x_{n}\right)$ with the unit of $S$, one can regard $p\left(a_{1}, \ldots, a_{n}\right)$ as an element of $M_{d}(S)$ in a natural way.

An atomic formula is an expression of the form $\left\|p\left(x_{1}, \ldots, x_{n}\right)\right\|$ for some degree 1 matrix *-polynomial. A positive positive quantifier-free formula $\varphi\left(x_{1}, \ldots, x_{n}\right)$ is an expression of the form

$$
f\left(\varphi_{1}\left(x_{1}, \ldots, x_{n}\right), \ldots, \varphi_{k}\left(x_{1}, \ldots, x_{n}\right)\right),
$$

where $f: \mathbb{R}^{n} \rightarrow \mathbb{R}$ is a continuous nondecreasing functions and $\varphi_{1}, \ldots, \varphi_{k}$ are atomic formulas. Given a positive quantifier-free formula $\varphi\left(x_{1}, \ldots, x_{n}\right)$, an operator system $S$, and elements $a_{1}, \ldots, a_{n}$ of $S$, one can define in a natural way the interpretation $\varphi\left(a_{1}, \ldots, a_{n}\right)$. We denote by $\operatorname{Ball}(S)$ the unit ball of the operator system $S$.

Definition 3.1. An operator system $S$ is positively existentially closed in the class of operator systems if for any operator system inclusion $S \subset T$, positive quantifier-free formula $\varphi\left(x_{1}, \ldots, x_{n}, y_{1}, \ldots, y_{m}\right)$, and $a_{1}, \ldots, a_{n} \in S$, one has that

$$
\inf \left\{\varphi\left(a_{1}, \ldots, a_{n}, b_{1}, \ldots, b_{m}\right): b_{1}, \ldots, b_{m} \in \operatorname{Ball}(T)\right\}=\inf \left\{\varphi\left(a_{1}, \ldots, a_{n}, b_{1}, \ldots, b_{m}\right): b_{1}, \ldots, b_{m} \in \operatorname{Ball}(S)\right\} .
$$

Equivalently, one can say that $S$ is positively existentially closed if for any operator system inclusion $S \subset T$, degree 1 matrix *-polynomials $p_{1}\left(x_{1}, \ldots, x_{n}, y_{1}, \ldots, y_{m}\right), \ldots, p_{k}\left(x_{1}, \ldots, x_{n}, y_{1}, \ldots, y_{m}\right)$, elements $a_{1}, \ldots, a_{n} \in$ $\operatorname{Ball}(S)$ and $b_{1}, \ldots, b_{m} \in \operatorname{Ball}(T)$, and $\varepsilon>0$, there exist $c_{1}, \ldots, c_{m} \in \operatorname{Ball}(S)$ such that, for every $i \in$ $\{1,2, \ldots, k\}$,

$$
\left\|p_{i}\left(a_{1}, \ldots, a_{n}, c_{1}, \ldots, c_{m}\right)\right\| \leq\left\|p_{i}\left(a_{1}, \ldots, a_{n}, b_{1}, \ldots, b_{m}\right)\right\|+\varepsilon
$$

We now observe that, for an operator system, being positively existentially closed is equivalent to having the weak expectation property. In the case of unital $\mathrm{C}^{*}$-algebras, this is proved in [29, Proposition 5.1].

Proposition 3.2. Suppose that $S$ is an operator system. The following statements are equivalent:

(1) $S$ has the weak expectation property;

(2) $S$ is positively existentially closed in the class of operator systems.

Proposition 3.2 generalizes [29, Proposition 4.18], where it is proved that for an exact operator system being positively existentially closed is equivalent to being nuclear.

In the following, we consider the construction of a $q$-minimal operator system from [52]. Suppose that $S$ is an operator system. Then the $q$-minimal operator system $\operatorname{OMIN}_{q}(S)$ is the image of $S$ under the direct sum of all the unital completely positive maps $\phi: S \rightarrow M_{q}(\mathbb{C})$. The canonical surjective isomorphism $\eta: S \rightarrow \mathrm{OMIN}_{q}(S)$ is a unital completely positive map such that $\eta_{q}: M_{q}(S) \rightarrow M_{q}\left(\mathrm{OMIN}_{q}(S)\right)$ is an order isomorphism. Observe that the assignment $S \mapsto \operatorname{OMIN}_{q}(S)$ is functorial, and any unital completely positive map $\phi: S \rightarrow T$ induces a canonical unital completely positive map $\operatorname{OMIN}_{q}(\phi): \operatorname{OMIN}_{q}(S) \rightarrow \operatorname{OMIN}_{q}(T)$. By injectivity of $M_{q}(\mathbb{C})$, if $\phi$ is a unital complete order embedding, then $\operatorname{OMIN}_{q}(\phi)$ is a unital complete order embedding. In particular, an inclusion of operator systems $S \subset T$ induces a canonical inclusion of operator systems $\operatorname{OMIN}_{q}(S) \subset \operatorname{OMIN}_{q}(T)$. Finally, $\operatorname{OMIN}_{q}\left(S^{* *}\right)$ is unitally completely order isomorphic to $\operatorname{OMIN}_{q}(S)^{* *}$, and if $\iota_{S}: S \rightarrow S^{* *}$ is the canonical inclusion map, then $\operatorname{OMIN}_{q}\left(\iota_{S}\right)$ can be identified with $\iota_{\operatorname{OMIN}_{q}(S)}$. We recall that an operator space $S$ is locally reflexive if for any finite-dimensional operator space $F$ and complete contraction $\phi: F \rightarrow S^{* *}, \phi$ is the point- $\sigma\left(S^{* *}, S^{*}\right)$-limit of completely contractions from $F$ to $S$; see [16, Section 4]. 
Proof of Proposition 3.2. The proof of [29, Proposition 4.18] shows that a positively existentially closed operator system satisfies Condition (2) of Proposition 2.3. Indeed, the existence of a unital completely positive map $\psi$ as in Condition (2) of Proposition 2.3 can be expressed in terms of the value of a suitable quantifier-free formula. We now prove the converse.

Suppose that $S$ is an operator system satisfying the weak expectation property. Suppose that $S \subset T$ is an operator system inclusion, $\varphi\left(x_{1}, \ldots, x_{n}, y\right)$ is a positive quantifier-free formula, $a_{1}, \ldots, a_{n} \in \operatorname{Ball}(S)$, $b_{1}, \ldots, b_{m} \in \operatorname{Ball}(T)$, and $\varepsilon>0$. We want to prove that there exist $c_{1}, \ldots, c_{m} \in \operatorname{Ball}(S)$ such that

$$
\varphi\left(a_{1}, \ldots, a_{n}, c_{1}, \ldots, c_{m}\right) \leq \varphi\left(a_{1}, \ldots, a_{n}, b_{1}, \ldots, b_{m}\right)+\varepsilon .
$$

Without loss of generality, we can assume that $T=B(H)$. Since $S$ satisfies the weak expectation property, there exists a unital completely positive map $\psi: B(H) \rightarrow S^{* *}$ such that $\left.\psi\right|_{S}$ is the inclusion map of $S$ into $S^{* *}$.

Set $F:=\operatorname{span}\left\{a_{1}, \ldots, a_{n}, b_{1}, \ldots, b_{m}, 1\right\} \subset B(H)$. One can choose $q \in \mathbb{N}$ large enough such that, denoting by $\eta: F \rightarrow \mathrm{OMIN}_{q}(F)$ the canonical linear isomorphism,

$$
\varphi\left(\eta\left(a_{1}\right), \ldots, \eta\left(a_{n}\right), \eta\left(b_{1}\right), \ldots, \eta\left(b_{m}\right)\right)=\varphi\left(a_{1}, \ldots, a_{n}, b_{1}, \ldots, b_{m}\right) .
$$

Consider now the unital completely positive map

$$
\operatorname{OMIN}_{q}(\psi): \operatorname{OMIN}_{q}(B(H)) \rightarrow \operatorname{OMIN}_{q}\left(S^{* *}\right) \cong \operatorname{OMIN}_{q}(S)^{* *}
$$

Observe that $\operatorname{OMIN}_{q}(B(H))$ is exact and, particularly, locally reflexive [17, Corollary 14.6.5]. Therefore $\mathrm{OMIN}_{q}(\psi)$ can be approximated in the point- $\sigma\left(\operatorname{OMIN}_{q}(S)^{* *}, \mathrm{OMIN}_{q}(S)^{*}\right)$-topology by completely contractive maps $\mathrm{OMIN}_{q}(F) \rightarrow \mathrm{OMIN}_{q}(S)$. Furthermore $\operatorname{OMIN}_{q}(B(H))$ is unitally completely order isomorphic to a direct sum of copies of $M_{q}(\mathbb{C})$. Fix $\delta>0$. A convexity argument as in the proof of Proposition 2.3 shows that there exists a unital completely positive map $\theta: \operatorname{OMIN}_{q}(F) \rightarrow \operatorname{OMIN}_{q}(S)$ such that $\left\|\left.\theta\right|_{\operatorname{OMIN}_{q}(E)}-\iota\right\|<\delta$, where $\iota: \mathrm{OMIN}_{q}(E) \rightarrow \mathrm{OMIN}_{q}(S)$ is the inclusion map. By choosing $\delta$ small enough one has that

$$
\begin{aligned}
\varphi\left(a_{1}, \ldots, a_{n},(\theta \circ \eta)\left(b_{1}\right), \ldots,(\theta \circ \eta)\left(b_{m}\right)\right) & \leq \varphi\left((\theta \circ \eta)\left(a_{1}\right), \ldots,(\theta \circ \eta)\left(a_{n}\right),(\theta \circ \eta)\left(b_{1}\right), \ldots,(\theta \circ \eta)\left(b_{m}\right)\right)+\varepsilon \\
& \leq \varphi\left(\eta\left(a_{1}\right), \ldots, \eta\left(a_{n}\right), \eta\left(b_{1}\right), \ldots, \eta\left(b_{m}\right)\right)+\varepsilon \\
& =\varphi\left(a_{1}, \ldots, a_{n}, b_{1}, \ldots, b_{m}\right)+\varepsilon .
\end{aligned}
$$

This concludes the proof that $S$ is positively existentially closed.

3.2. The weak expectation property and the complete tight Riesz interpolation. The complete tight Riesz interpolation property has been introduced for unital $\mathrm{C}^{*}$-algebras by Kavruk in [35]. We consider here its straightforward generalization to operator systems. Let $S \subset T$ be an inclusion of operator systems. For elements $a, b$ of $M_{n}(T)$, write $a \ll b$ if $b-a \geq \delta 1$ for some $\delta>0$.

Definition 3.3 (Kavruk). The operator system $S$ has the relative complete tight Riesz interpolation property in $T$ if for any $n, k \in \mathbb{N}, x_{1}, \ldots, x_{k}, y_{1}, \ldots, y_{k} \in M_{d}(S)_{\text {sa }}$ and $b \in M_{n}(T)_{\text {sa }}$ such that $x_{i} \ll b \ll y_{j}$ for every $i, j \in\{1,2, \ldots, k\}$, there exists $a \in M_{n}(S)_{\text {sa }}$ such that $x_{i} \leq a \leq y_{j}$ for every $i, j \in\{1,2, \ldots, k\}$.

It is proved in [35, Theorem 7.4] that a unital $\mathrm{C}^{*}$-algebra $A \subset B(H)$ satisfies the weak expectation property if and only if it has the relative complete tight Riesz interpolation property in $B(H)$. An alternative proof using the characterization of the model-theoretic characterization of the weak expectation property is presented in [29, Theorem 5.5]. In view of Proposition 3.2, such a proof applies equally well to operator systems, and it gives the following.

Proposition 3.4. Suppose that $S \subset B(H)$ is an operator system. The following assertions are equivalent.

(1) $S$ satisfies the weak expectation property;

(2) $S$ has the relative complete tight Riesz interpolation property in $B(H)$;

(3) $S$ has the relative complete tight Riesz interpolation property in $T$ for some operator system inclusion $S \subset T$, where $T$ is an operator system with the weak expectation property.

\section{REFERENCES}

1. Erik M. Alfsen, Compact convex sets and boundary integrals, Springer-Verlag, New York-Heidelberg, 1971.

2. William Arveson, Subalgebras of $C^{*}$-algebras, Acta Mathematica 123 (1969), no. 1, 141-224.

3. Subalgebras of $C^{*}$-algebras II, Acta Mathematica 128 (1972), no. 1, 271-308.

4. no. $4,1065-1084$. 
5. — The noncommutative Choquet boundary III: operator systems in matrix algebras, Mathematica Scandinavica 106 (2010), no. 2, 196-210.

6. — The noncommutative Choquet boundary II: hyperrigidity, Israel Journal of Mathematics 184 (2011), no. 1, 349-385.

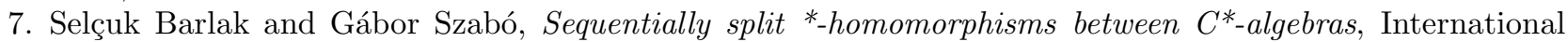
Journal of Mathematics 27 (2016), no. 13, 1650105, 48.

8. Selçuk Barlak, Gábor Szabó, and Christian Voigt, The spatial Rokhlin property for actions of compact quantum groups, Journal of Functional Analysis 272 (2017), no. 6, 2308-2360.

9. Nathanial P. Brown and Narutaka Ozawa, $C^{*}$-algebras and finite-dimensional approximations, Graduate Studies in Mathematics, vol. 88, American Mathematical Society, Providence, RI, 2008.

10. Man-Duen Choi and Edward G. Effros, The completely positive lifting problem for $C^{*}$-algebras, Annals of Mathematics 104 (1976), no. 3, 585-609.

11. Injectivity and operator spaces, Journal of Functional Analysis 24 (1977), no. 2, 156-209.

12. Man Duen Choi and Edward G. Effros, Lifting problems and the cohomology of $C^{*}$-algebras, Canadian Journal of Mathematics 29 (1977), no. 5, 1092-1111.

13. Man-Duen Choi and Edward G. Effros, Nuclear $C^{*}$-algebras and the approximation property, American Journal of Mathematics 100 (1978), no. 1, 61-79.

14. Kenneth R. Davidson and Matthew Kennedy, The Choquet boundary of an operator system, Duke Mathematical Journal 164 (2015), no. 15, 2989-3004.

15. Edward G. Effros, Aspects of noncommutative order, $\mathrm{C}^{*}$-algebras and applications to physics (Proc. Second Japan-USA Sem., Los Angeles, Calif., 1977), Lecture Notes in Mathematics, vol. 650, Springer, Berlin, 1978, pp. 1-40.

16. Edward G. Effros, Narutaka Ozawa, and Zhong-Jin Ruan, On injectivity and nuclearity for operator spaces, Duke Mathematical Journal 110 (2001), no. 3, 489-521.

17. Edward G. Effros and Zhong-Jin Ruan, Operator spaces, London Mathematical Society Monographs. New Series, vol. 23, Oxford University Press, 2000.

18. Edward G. Effros and Corran Webster, Operator analogues of locally convex spaces, Operator algebras and applications (Samos, 1996), NATO Adv. Sci. Inst. Ser. C Math. Phys. Sci., vol. 495, Kluwer Acad. Publ., Dordrecht, 1997, pp. 163-207.

19. Douglas Farenick, Ali S. Kavruk, Vern I. Paulsen, and Ivan G. Todorov, Characterisations of the weak expectation property, arXiv:1307.1055 (2013).

20. 207-238.

21. Douglas Farenick and Phillip Morenz, $C^{*}$-extreme points in the generalized state spaces of a $C^{*}$-algebra, Transactions of the American Mathematical Society 349 (1997), no. 5, 1725-1748.

22. Douglas Farenick and Phillip B. Morenz, $C^{*}$-extreme points of some compact $C^{*}$-convex sets, Proceedings of the American Mathematical Society 118 (1993), no. 3, 765-775.

23. Douglas Farenick and Vern I. Paulsen, Operator system quotients of matrix algebras and their tensor products, Mathematica Scandinavica 111 (2012), no. 2, 210-243.

24. Douglas R. Farenick, Extremal matrix states on operator systems, Journal of the London Mathematical Society 61 (2000), no. 3, 885-892.

25. - Pure matrix states on operator systems, Linear Algebra and its Applications 393 (2004), $149-173$.

26. Eusebio Gardella, Mehrdad Kalantar, and Martino Lupini, Rokhlin dimension for compact quantum group actions, arXiv:1703.10999 (2017).

27. Eusebio Gardella and Martino Lupini, Equivariant logic and applications to $C^{*}$-dynamics, arXiv:1608.05532 (2016).

28. Isaac Goldbring and Martino Lupini, Model-theoretic aspects of the Gurarij operator system, Isreal Journal of Mathematics, in press.

29. Isaac Goldbring and Thomas Sinclair, Omitting types in operator systems, Indiana University Mathematics Journal, to appear.

30. __ On Kirchberg's embedding problem, Journal of Functional Analysis 269 (2015), no. 1, $155-198$.

31. Kyung Hoon Han and Vern I. Paulsen, An approximation theorem for nuclear operator systems, Journal of Functional Analysis 261 (2011), no. 4, 999-1009.

32. Alan Hopenwasser, Robert L. Moore, and Vern I. Paulsen, $C^{*}$-extreme points, Transactions of the American Mathematical Society 266 (1981), no. 1, 291-307. 
33. Marius Junge and Gilles Pisier, Bilinear forms on exact operator spaces and $B(H) \otimes B(H)$, Geometric and Functional Analysis 5 (1995), no. 2, 329-363.

34. Ali Kavruk, Vern I. Paulsen, Ivan G. Todorov, and Mark Tomforde, Tensor products of operator systems, Journal of Functional Analysis 261 (2011), no. 2, 267-299.

35. Ali S. Kavruk, The weak expectation property and Riesz interpolation, arXiv:1201.5414 (2012).

36. Ali S. Kavruk, Vern I. Paulsen, Ivan G. Todorov, and Mark Tomforde, Quotients, exactness, and nuclearity in the operator system category, Advances in Mathematics 235 (2013), 321-360.

37. Ali Samil Kavruk, Nuclearity related properties in operator systems, Journal of Operator Theory 71 (2014), no. 1, 95-156.

38. Aldo J. Lazar, Spaces of affine continuous functions on simplexes, Transactions of the American Mathematical Society 134 (1968), no. 3, 503-525.

39. Richard I. Loebl and Vern I. Paulsen, Some remarks on $C^{*}$-convexity, Linear Algebra and its Applications 35 (1981), 63-78.

40. Martino Lupini, Frä̈sé limits in functional analysis, arXiv:1510.05188 (2015).

41. Vern I. Paulsen, Completely bounded maps on $C^{*}$-algebras and invariant operator ranges, Proceedings of the American Mathematical Society 86 (1982), no. 1, 91-96.

42. Completely bounded homomorphisms of operator algebras, Proceedings of the American Mathematical Society 92 (1984), no. 2, 225-228.

43. - Completely bounded maps and operator algebras, Cambridge Studies in Advanced Mathematics, vol. 78, Cambridge University Press, Cambridge, 2002.

44. Gilles Pisier, Introduction to operator space theory, London Mathematical Society Lecture Note Series, vol. 294, Cambridge University Press, Cambridge, 2003.

45. Lothar M. Schmitt, Characterization of $L^{2}(\mathcal{M})$ for injective $W^{*}$-algebras $\mathcal{M}$, Mathematica Scandinavica 57 (1985), no. 2, 267-280.

46. Lothar M. Schmitt and Gerd Wittstock, Characterization of matrix-ordered standard forms of $W^{*}$-algebras, Mathematica Scandinavica 51 (1982), no. 2, 241-260.

47. Corran Webster and Soren Winkler, The Krein-Milman theorem in operator convexity, Transactions of the American Mathematical Society 351 (1999), no. 1, 307-322.

48. Soren Winkler, The non-commutative Legendre-Fenchel transform, Mathematica Scandinavica 85 (1999), no. 1, 30-48.

49. Gerd Wittstock, Ein operatorwertiger Hahn-Banach Satz, Journal of Functional Analysis 40 (1981), no. 2, 127-150.

50. _ Extension of completely bounded $C^{*}$-module homomorphisms, Operator algebras and group representations, Vol. II (Neptun, 1980), Monogr. Stud. Math., vol. 18, Pitman, Boston, MA, 1984, pp. $238-250$.

51. _ On matrix order and convexity, Functional analysis: surveys and recent results, III (Paderborn, 1983), North-Holland Math. Stud., vol. 90, North-Holland, Amsterdam, 1984, pp. 175-188.

52. Blerina Xhabli, The super operator system structures and their applications in quantum entanglement theory, Journal of Functional Analysis 262 (2012), no. 4, 1466-1497.

Mathematics Department, California Institute of Technology, 1200 E. California Blvd, MC 253-37, Pasadena, Ca 91125

E-mail address: lupini@caltech.edu

URL: http://www.lupini.org/ 